\title{
Demography and Cultural Innovation: a Model and its Implications for the Emergence of Modern Human Culture
}

\author{
Stephen Shennan
}

\begin{abstract}
In recent years there has been a major growth of interest in exploring the analogies between the genetic transmission of information from one generation to the next and the processes of cultural transmission, in an attempt to obtain a greater understanding of how culture change occurs. This article uses computer simulation to explore the implications of a specific model of the relationship between demography and innovation within an evolutionary framework. The consequences of innovation appear far more successful in larger populations than in smaller ones. In conclusion, it is suggested that the model has major implications for the origins of modern human culture in the last 50,000 years, which may be seen not as the result of genetic mutations leading to improved cognitive capacities of individuals, but as a population consequence of the demographic growth and increased contact range which are evident at this time. It is also proposed that the model may be of general relevance for understanding the process of cultural evolution in modern and pre-modern humans.
\end{abstract}

The processes of cultural innovation and transmission are analogous in many respects to genetic mutation and transmission (Cavalli-Sforza \& Feldman 1981; Boyd \& Richerson 1985). Some genetic mutations produce deleterious effects on fitness and others produce beneficial ones. Where individuals are at high levels of fitness, mutations are likely to be deleterious; where they are much less fit, mutations are much more likely to be beneficial. A substantial proportion of mutations, however, will only make a slight difference one way or the other. It seems at least plausible that cultural innovations operate in a similar manner to genetic mutations. Some are likely to have a positive effect on the biological fitness of their bearers, others a negative effect, while the majority will make little difference. In the case of cultural innovations, however, the situation is rather different. Because cultural traits can be transmitted obliquely (by non-parents of the parental generation) and horizontally (between peers), independently of genes, we can also talk about the desirability of innovations in terms of their attractiveness to oth- ers, without committing ourselves one way or the other as to their effect on biological fitness. Nevertheless, the idea that some innovations will be less attractive to imitate and others more so, while some make only a marginal difference to existing options, seems a reasonable one.

The specific aim of this article is to explore the consequences of innovations in small populations of the kind that prevailed in the world until the advent of agriculture, and which must have provided the context for the evolution of human culture. The model presented here focuses on the processes involved in the transmission and change of craft traditions, such as stone tool production. It is primarily innovation in such traditions that defines the cultural expansions of the last 50,000 years and makes them an archaeologically visible phenomenon. This focus on craft traditions has other implications, however, since it is increasingly clear that the transmission of craft traditions, as opposed to other kinds of information, has its own specific characteristics. By and large, in traditional societies craft traditions are passed on 
from a parent to the same gender offspring once a generation during childhood (Shennan \& Steele 1999); they are only very rarely learned from peers although they may be learned from same gender adults who are not parents. Any given craft will have a variety of different aspects which may be susceptible to innovation and at least some of which have greater or lesser biological fitness consequences, and/or greater or lesser attractiveness as models to be imitated, whether or not this attractiveness has any bearing on biological fitness (see below for a discussion of this issue). In a traditional society, men and women are likely to practise a variety of different crafts. Thus, any individual will have a range of cultural attributes that are potentially subject to the cultural equivalent of fitness-altering mutations, in the sense that they have an effect on the biological fitness consequences of the attribute in question and/or on its attractiveness as a trait to be imitated.

\section{The model}

The model of cultural evolution used here is adapted from one developed by Peck and colleagues (Peck et al. 1997) to explore the relative benefits of sexual and asexual reproduction. ${ }^{1}$ These authors constructed a model of mutation based on the ideas of R.A. Fisher (1930). The model allows for both beneficial and deleterious mutations, and mutant effects are biased so that there is a correlation between the state of an allele prior to mutation and its post-mutation state. Furthermore, in this particular version of the Fisherian mutation model, a substantial fraction of mutations produce a very small change in fitness.

The aim here is to use Peck et al.'s model to explore the effect of cultural 'mutations' (innovations) on the fitness and cultural attractiveness of human populations, in relation to the size of those populations. The model required two main modifications in order to make it appropriate for modelling cultural evolution. First, the transmission process was altered so that 'haploid' transmission from one 'cultural parent' to one 'cultural offspring' was the only possibility, in contrast to the diploid transmission of sexual reproduction. Second, it was modified so that the cultural parent need not be the biological parent, in other words, so that transmission could be oblique.

The oblique transmission model and its results are described further below. The initial model was based solely on haploid, one parent to one offspring, inheritance.

\section{The simple model}

Let $L$ represent the number of craft-technique attributes possessed by an individual that are potentially subject to the cultural equivalent of fitness-altering mutations. That is, $L$ is the number of attributes or traits of an individual's set of techniques that are subject to selection. A multiplicative model of fitness determination is assumed, which makes it possible to assign a selection coefficient to each possible alternative technique (or attribute state). In this simple version of the model without oblique transmission, selection refers specifically to biological natural selection, since the only way in which a specific technique can increase or decrease in frequency is through its effect on the reproductive success of its bearer.

It is assumed that the state of each of an individual's $L$ attributes can be represented by a real number $x$, where

$$
-\infty<x<+\infty
$$

It is assumed further that the optimum state for each attribute is achieved when $x=0$. Let $d_{i, j}$ represent the absolute value of the deviation from the optimum state of the version of the $i^{\text {th }}$ attribute in the $j^{\text {th }}$ adult for a particular generation (i.e. $d_{i j}=|x|$ ). Let $D_{j}$ represent the sum of these deviations for the $j^{\text {th }}$ adult. That is:

$$
D_{j}=\sum_{i=1}^{L} d_{i j}
$$

Let $w_{j}$ represent the fertility (and thus the fitness) of the $j^{\text {th }}$ adult during a particular generation, relative to an adult with the notional optimum set of techniques (This is one for which $x=0$ for all $L$ attributes under selection). The value of $w_{j}$ is assumed to depend on $D_{j}$ as follows:

$$
w_{j}=\exp \left(-D_{j}\right)
$$

To make the multiplicative nature of the model more apparent, this can be re-written as:

$$
w_{j}=\prod_{i=1}^{L}\left[\exp \left(-d_{i j}\right)\right]
$$

The model starts with a perfectly fit population. Cultural transmission occurs, with the parental value of a cultural trait being passed on to its offspring. In the course of transmission innovations can occur, which can be either beneficial or deleterious. The probability that any given attribute state adopted by an offspring will have just undergone an innovation is represented by $\mu$, the attribute innovation rate. An 
innovation is assumed to change the value associated with an attribute by an amount $m$, such that the innovated value of the attribute is given by $x=m+$ $x^{*}$, where $x^{*}$ is the value of $x$ that would have been associated with the attribute if no innovation had occurred (i.e. $x^{*}$ is the parental value of $x$ for the attribute in question).

The effect of the innovations on fitness is modelled by a 'reflected gamma' distribution with shape parameter $1 / 2$. As Figure 1 shows, the shape of the distribution is such that the majority of innovations have very little effect, while some have a significant one. The probability density function $(f(y))$ for the reflected gamma distribution used in this article is given by:

$$
f(y)=\frac{a^{1 / 2} e^{-a|y|}|y|^{-1 / 2}}{2 \Gamma(1 / 2)}
$$

The entire distribution is symmetrical about zero, so the expected value of $m$ is zero. For the multiplicative model was set at $a$ 24.253. This ensures that innovations to near-perfect attributes will decrease fitness by about 2 per cent, and that the coefficient of variation in fitness is about 18 per cent.

Any innovation that causes the trait value to move away from zero, whether in a positive or negative direction, is deleterious. Under the above set of assumptions, innovations are bound to be deleterious initially, but as the process of transmission proceeds and the population becomes less fit, the probability that the innovations will be beneficial increases. In other words, there is a greater probability that an innovation will move the trait value towards zero rather than away from it. Eventually the process reaches a stationary distribution, with beneficial and deleterious mutations having equal effects. A mean and standard deviation for the fitness value are obtained from a given number of generations once stationarity has been reached. These fitnesses can be compared for various combinations of the parameters of interest.

\section{Parameter values}

Unfortunately, there is very little to go on as far as specifying parameter values for modelling cultural evolution is concerned. In the case of the fitness / attractiveness effect of innovations, Peck et al.'s (1997) value has been followed. This is based on genetic data which suggest that mutations decreasing fitness by 2 per cent are common. Accordingly, the distribution of 'fitness' effects is parameterized so that the average effect of a 'mutation' to a 'perfect' trait is to decrease 'fitness' by 2 per cent.

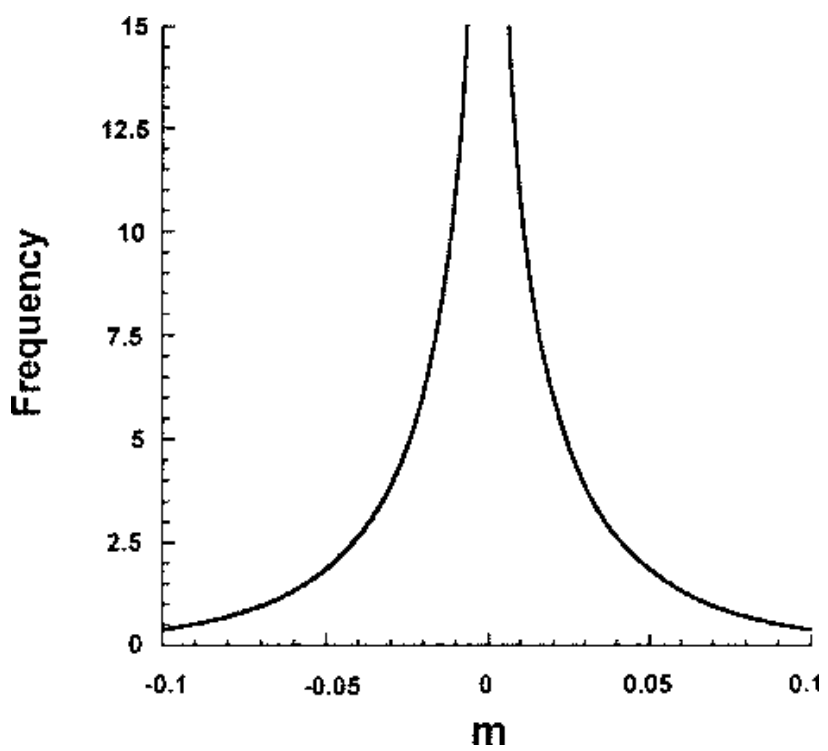

Figure 1. The distribution of $\mathrm{m}$ used in the simulation studies. This is a reflected gamma distribution (see text). The value of a is set to 24.253. This ensures that the average selection coefficient associated with a perfect allele $(\mathrm{x}=0)$ that has undergone a single mutation is $\mathrm{s}=0.02$.

Similarly, the number of craft attributes $(L)$ capable of being affected by fitness-altering innovations is unclear. Taken across the range of crafts that an individual would have had to learn (cf. Hewlett \& Cavalli-Sforza 1986), however, the lower limit seems unlikely to have been very small. An upper limit is much harder to set. We have arbitrarily selected a value of 100 for the purposes of this article. Experimentation with other values produced qualitatively identical results.

Next we have to consider the value of $\mu$, the attribute state innovation rate. Biologically realistic ones for genetic mutations lie in the range $10^{-9}-10^{-5}$. Rates of innovation in cultural traits relating to craft traditions, however, are unknown. We have taken the view that they are likely to be markedly higher than those for genetic mutations and have experimented with rates of $0.01,0.05$ and 0.1 . In other words, to take the last of these as an example, we have assumed that there is a 1 in 10 probability that a mutation will occur in a trait per generation. Thus, if there are 100 attributes, an individual will be characterized by an average of 10 innovations per generation.

Since the object of the simulations is to investigate the effect of population size on the extent to which cultural innovations are advantageous, the simulations run the model for different population 
sizes and look at the effect on the mean fitness of population members. The populations concerned are envisaged as groups of regularly interacting individuals. The size of the population was varied from 5 to 105 in increments of 10 . As we show below, population effects had tapered off considerably by the time the 105 value had been reached (although the precise point at which they will taper off is a function of the number of attributes - the greater the number of attributes, the higher it will be: Peck pers. comm.).

It is extremely important to understand that we are not talking about the total number of individuals in the interacting group, $N$, when we refer to the population size, but to a notional value of roughly $N / 4$, which may be considered to represent the effective population size. That is to say, population size here refers to the number of individuals in a given generation who are 'cultural parents' in the sense described above: either mothers passing on their craft techniques to their daughters, or fathers passing on their techniques to their sons. If we assume an equal ratio for the sexes and that roughly 50 per cent of the population at any given time are subadults, then an $N / 4$ value of 5 corresponds to a population size of 20, which is, if anything, rather large for the size of a minimal hunter-gatherer group.

\section{Initialization, data collection and descriptive statistics}

Simulation trials were initialized by generating an effective population of $N / 4$ individuals (where $N$ refers to the total number of individuals, adults and children of both genders, in the population), each of which had a 'perfect' set of cultural traits. For each individual in each generation the values of its attributes are reproduced with a certain probability of innovation taking place. As noted above, because the population starts off at perfect 'fitness', the initial effect of all innovations is deleterious and the population fitness decreases, but eventually stationarity is reached for a given combination of parameters. Before the main simulations were carried out, trials were undertaken to establish the number of generations required to reach stationarity; this varied depending on the precise combination of parameters. The initial non-stationary population 'fitness' values were discarded and 'fitness' statistics only calculated for the last $n$ thousand equilibrium generations.

The key statistics of interest to us concern fitness. Mean fitness is represented by $\bar{w}$. The value of $\bar{w}$ for a particular generation was calculated by averaging the $w_{j}$ values over all the $N / 4$ adults present during that generation (i.e. in terms of our model of same gender transmission, the number of adults of a given gender). Thus we have $0<\bar{w}<1$.

Despite the lack of directional change once stationarity has been reached there is still considerable variation over time from one generation to the next, in $\bar{w}$, especially for the small population sizes. Therefore we calculated $\overline{\bar{w}}_{g}$ : the geometric mean of the $\bar{w}$ values for those generations of the trial after stationarity had been reached. The geometric mean is preferable in this case because, unlike the arithmetic mean, it will always give values which scale consistently with reproductive success (see Peck et al. 1997).

\section{Results}

The results of the simple parental transmission model for mutation rates of $0.01,0.05$ and 0.1 are shown in Figure $2 \mathrm{a}-\mathrm{c}$. It is apparent that there is a major increase in culturally-based mean biological fitness as population size increases. Between $N / 4=5$ and N/4 $=50$, where the increase in fitness begins to level out, the equilibrium geometric mean fitness value of the population increases by around four orders of magnitude, or 10,000-fold, even though the population size increase across this range is only 10 -fold. Of course, the increase is greatest in the range $N / 4=5$ to $N / 4=15$ (corresponding to group sizes of 20-60 on the assumptions made above). Over this range the geometric mean fitness of the population increases by around 1000-fold. From N/4 25 to N/4 $=85$ the rate of increase in geometric mean fitness with increasing population size is linear and much lower, but still results in a $4-5$ fold increase over the range.

This is true for all three mutation rates selected. In other words, in larger populations there is a far greater probability of fitness-enhancing innovations being maintained in the population and deleterious ones being deleted, entirely as a result of the decreasing role of sampling effects as populations get bigger.

\section{The modified model: introducing oblique transmission} It was suggested earlier, following Shennan \& Steele (1999), that although craft activities in traditional societies are generally learned by children/adolescents from the same gender parent, sometimes they are learned instead from other adults. The object of this modified version of the model is to explore the implications of this fact for the relationship between population size and culturally-based biological fitness derived from the initial model. The introduction of 

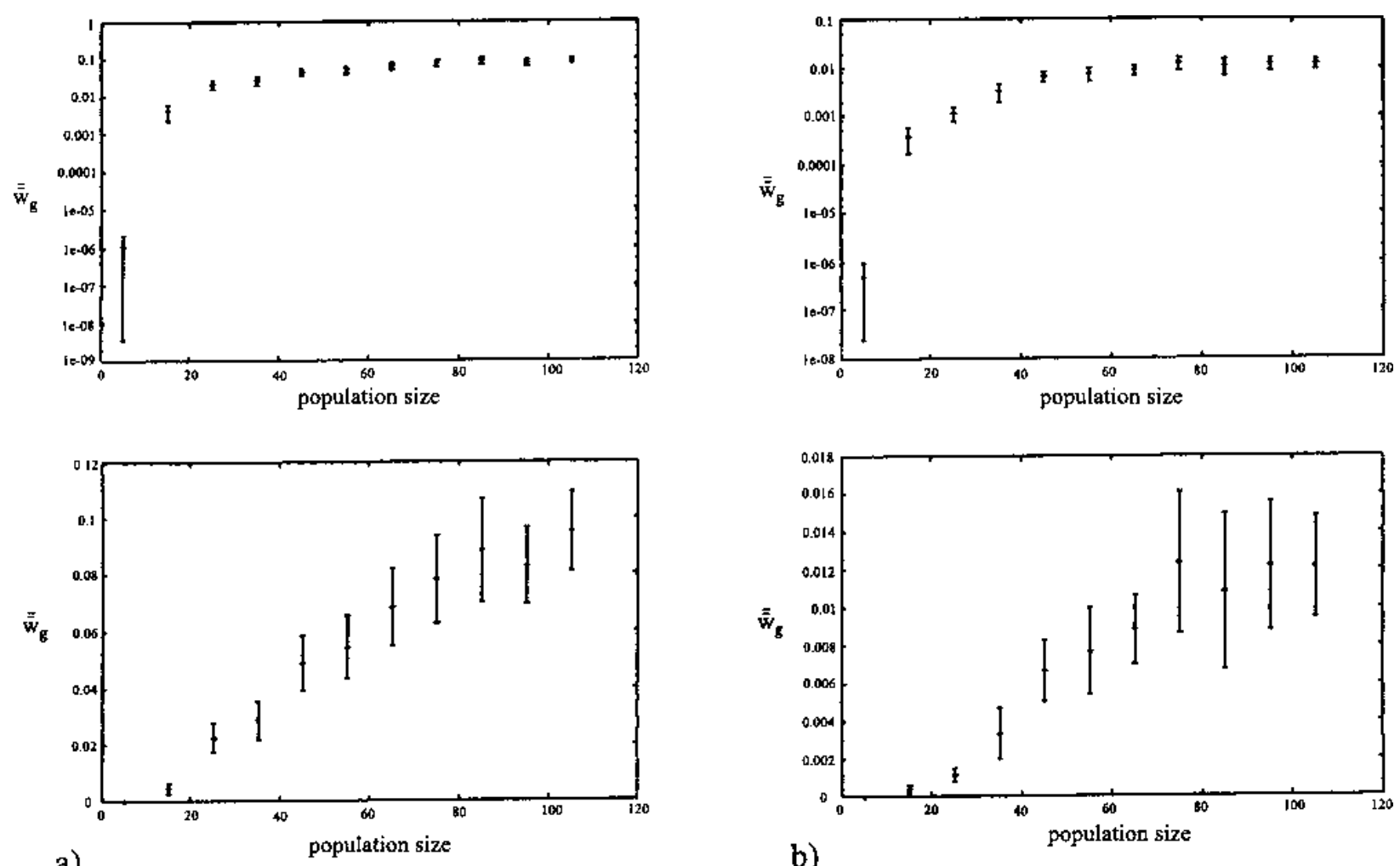

a)

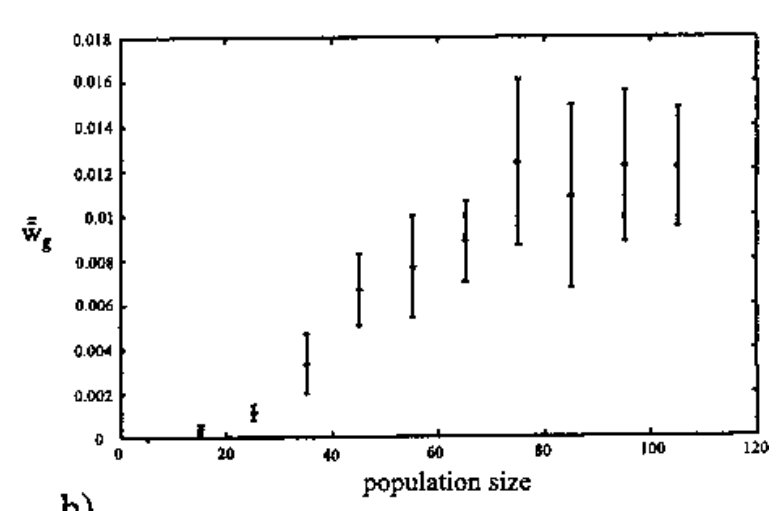

b)
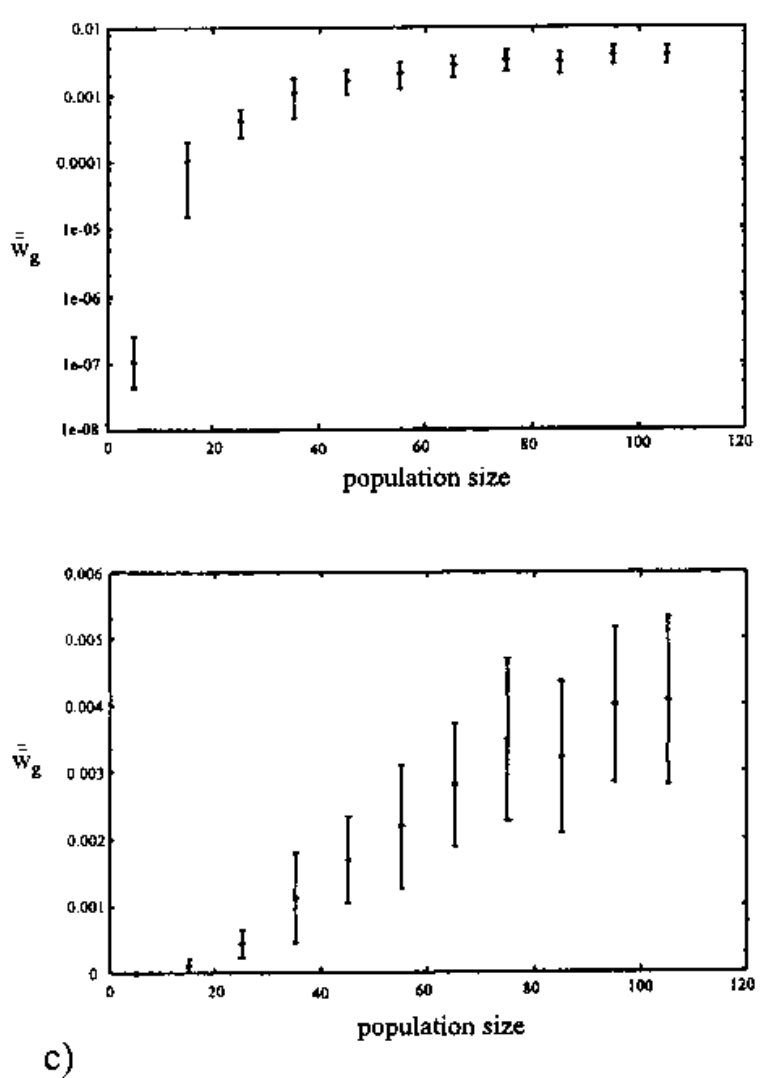

Figure 2. The results of the simulations using the simple model of one parent to offspring inheritance without oblique transmission. The horizontal axis represents effective population size, defined here as $\mathrm{N} / 4$, where $\mathrm{N}$ is total population size (see text). The vertical axis represents population geometric mean fitness. For all simulations $\mathrm{L}$ (the number of craft attributes) = 100; a (the gamma distribution parameter) $=24.253$ (see Fig. 1). $2 a-c$ Innovation rates $0.01,0.05$ and 0.1 respectively; above, $\log$ scale; below, linear scale.

oblique transmission raises a major substantive issue. In the simple model there is a direct link between biological and cultural success. Since cultural attributes are only transmitted from parents to offspring, the only way that a specific cultural trait will spread through a population is if its bearers are reproductively more successful than others; hence 'fitness' can be interpreted in the strict biological sense of Peck et al.'s original model. The way in which the terms of the model translate into a situation where this link is broken, or at least weakened, must now be considered.

Let us imagine a case where an individual does not learn from their biological parent but from a randomly selected nearby adult, or from that adult 
among those nearby who seems most desirable in some respect as a model. In such cases the 'cultural fitness' of an adult individual with a certain set of traits will be based on a combination of their reproductive success (to the extent that parent-offspring cultural transmission continues to play an important role), and their attractiveness as a model for imitation. Boyd \& Richerson's (1985) concepts of directly and indirectly biased cultural transmission provide a basis for conceptualizing variation in attractiveness in this context. In directly-biased transmission, individuals actively compare the way a number of potential models carry out some activity and opt for the one they consider the best; for example, the individual with what they perceive to be the most effective lithic-knapping technique. Indirectly-biased transmission describes the case where individuals take someone as a model in some activity or practice because they regard that person as generally prestigious in some way and thus as a model to be followed. In both cases the criteria on which individuals select models may well be connected with reproductive success because of the nature of human evolved psychologies; since transmission is oblique, however, they do not necessarily have to be.

As innovations occur, there is no guarantee that they will be found attractive by others; some will prove to be less attractive and others more so, while others again are considered only marginally more or less attractive than the existing options. In other words, our $\overline{\bar{w}}_{g}$ values are no longer simply the mean biological fitness values of our populations. They represent a combination of this and the mean attractiveness of members of the population as models to imitate. Again, we can investigate the way in which this combined measure of biological fitness and attractiveness $\overline{\bar{w}}_{g}$ changes with population size.

\section{Specifying the modified model}

To take account of oblique transmission, the simple model just described was modified by introducing two new parameters. The first $(z)$ specifies, for a given trait, the probability that it will not be inherited from the same gender parent. If $z>0$, then the individual chosen as the model is the individual with the highest fitness / attractiveness value for that trait of the $k$ randomly chosen individuals specified by the second parameter $(k)$. Where $k=1$ this reduces to random selection; where $k=N / 4-1$ it corresponds to the most attractive individual of the same gender in the adult generation in the population as a whole (excluding the parent). It seems reasonable to suggest that plausible $k$ values are likely to be low, with individuals inspecting the small number of available examples in the immediate vicinity.

To select the most attractive member of the pool with respect to a specific trait in this way is to enable non-parental oblique transmission to give the maximum possible benefit to the members of the population. In a real situation it is highly unlikely that an individual would always be able to pick out the most beneficial older individual to copy; after all, this is why unreflecting imitation is so widely prevalent in human culture (see again Boyd \& Richerson 1985). Empirically realistic results would therefore be likely to fall somewhere in between those of the simple model described above and those of the modified model to be described below.

\section{Results}

Figure 3a-d shows the results of running the simulation for various values of the oblique transmission rate, $z=0.05,0.2,0.4,0.6$. That is to say any given trait will be taken from a non-parent 5 per cent, 20 per cent, etc., of the time. In each case, the nonparental individual whose trait is copied is the individual with the highest fitness/attractiveness value for that trait, out of a randomly selected pool of five individuals. The results show the same pattern as in the case where transmission is solely from parent to offspring. As population size of the interacting group increases, the mean combined fitness/attractiveness of the population increases; very markedly when the population is small, less rapidly as the absolute population size becomes larger. The rate of change also varies, however, with the oblique transmission rate. For $z=0.05$ (Fig. 3a), when a given trait is copied from a non-parent 5 times out of 100 on average, the population mean fitness / attractiveness increases by some three orders of magnitude (1000-fold) over the range $N / 4=5$ to $N / 4=25$ (i.e. for a total population size of $c$. 20-100), and thereafter increases by around five times over the range $N / 4=25$ to $N / 4=75$. For $z$

Figure 3. The results of the simulations using the modified model of one parent to offspring inheritance with oblique transmission. The horizontal axis represents effective population size, defined here as $\mathrm{N} / 4$, where $\mathrm{N}$ is total population size (see text). The vertical axis represents population geometric mean fitness $\left(\overline{\mathrm{w}}_{\mathrm{g}}\right)$. For all simulations $\mathrm{L}$ (the number of craft attributes) $=$ 100; a (the gamma distribution parameter) $=24.253$ (see Fig. 1); $\mathrm{m}$ (the innovation rate) $=0.05$. 3a-d Oblique transmission rates of $0.05,0.2,0.4$ and 0.6 respectively; above, log scale; below linear scale. 

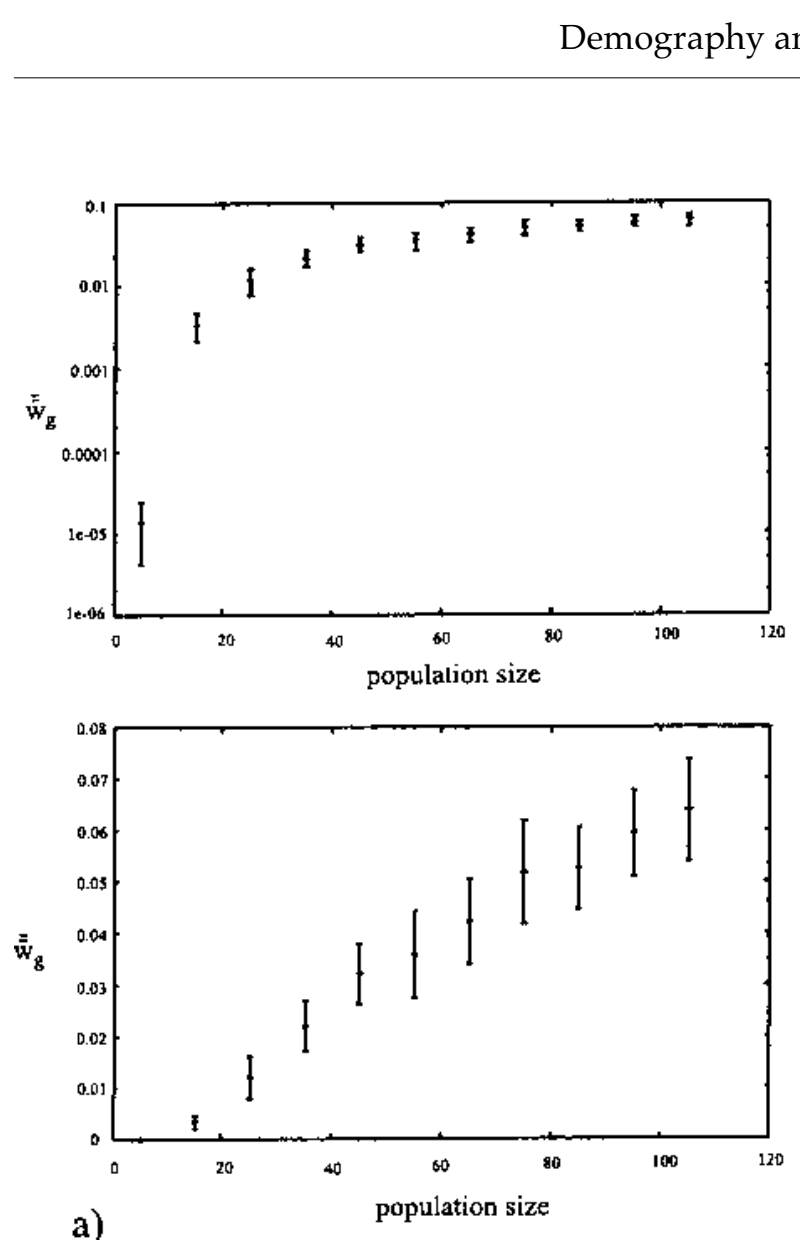

a)
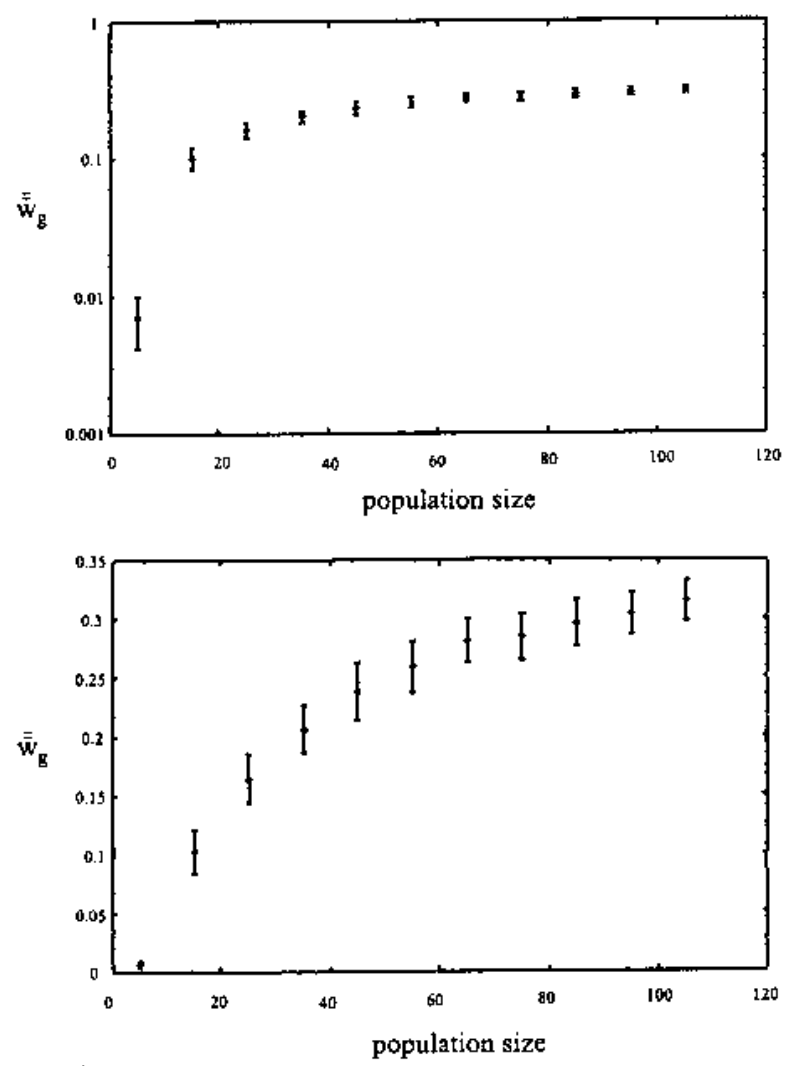

c)
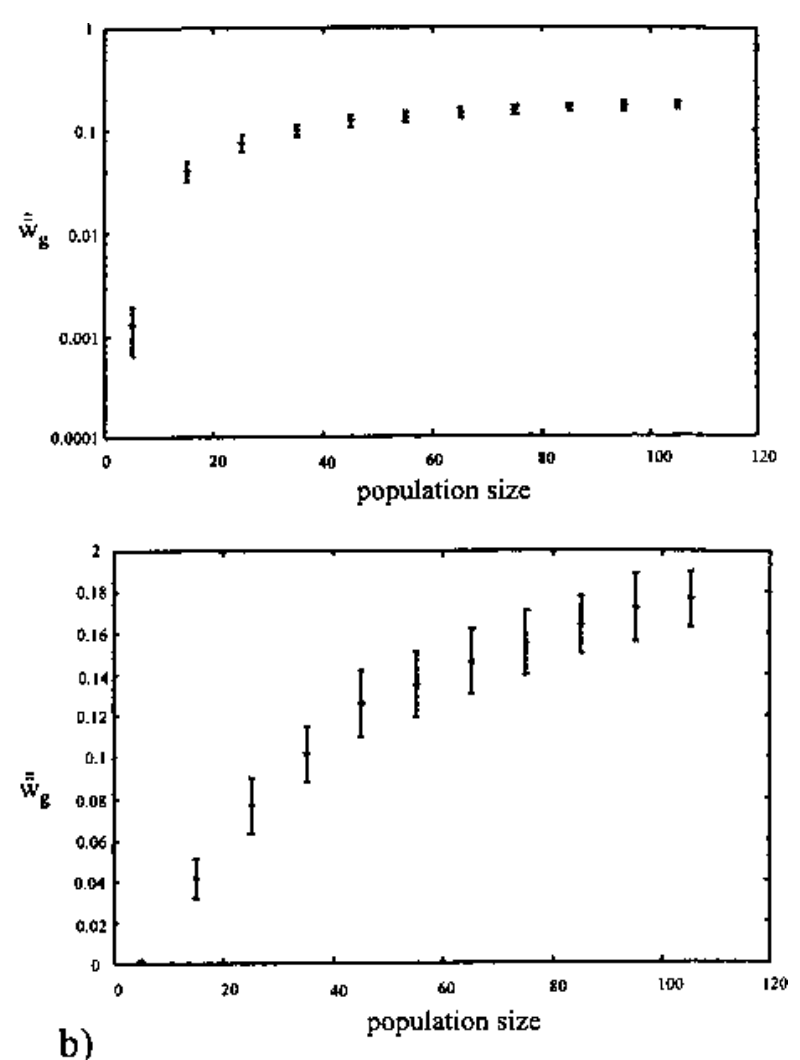

b)
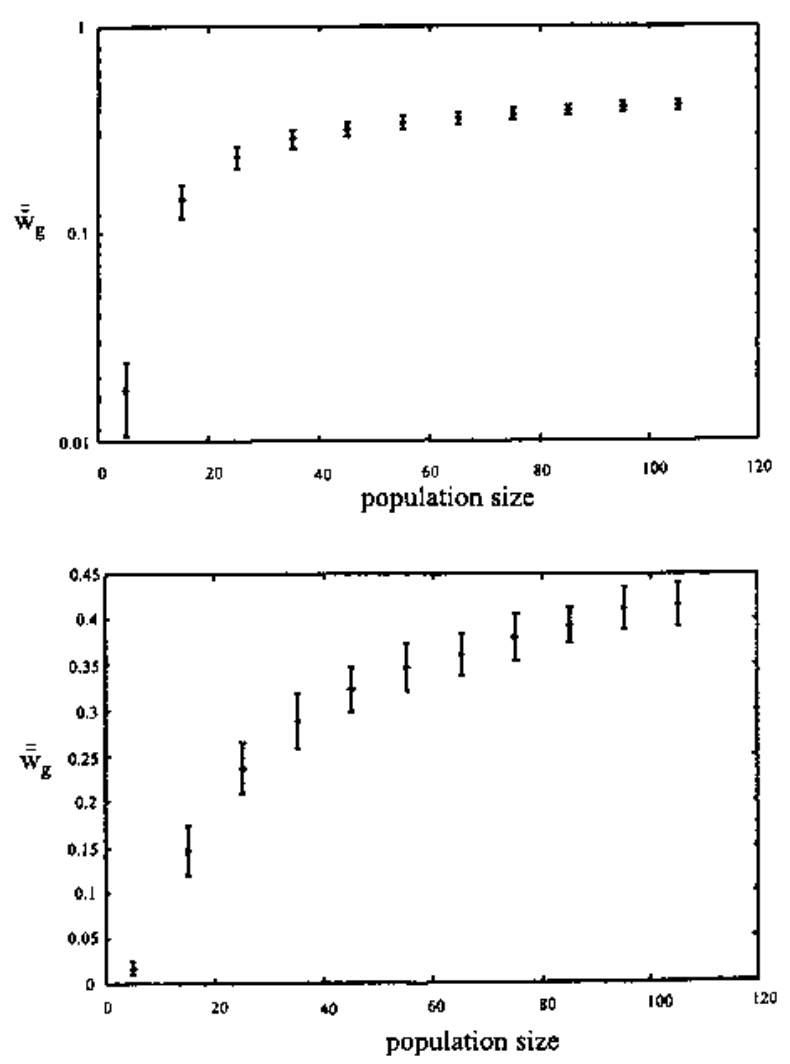

d)

11 
$=0.2$ (Fig. $3 \mathrm{~b}$ ), the corresponding values are approximately two orders of magnitude (100-fold) and twofold. For values of $z=0.4$ and 0.6 (Fig. 3c-d) the corresponding values are ten-fold and two-fold. In other words, even though the effect is markedly attenuated as the rate of oblique transmission increases, increasing population size still makes a very big difference to the mean fitness/attractiveness of the population, for the same sampling effect reasons as in the simple parental transmission case. As noted above, the cases cited in Shennan \& Steele (1999; and see also Hewlett \& Cavalli-Sforza 1986) suggest that, in general, oblique transmission rates of craft skills are relatively low, certainly below $z=0.4$. It is also worth pointing out that oblique transmission of traits produces its greatest gains in fitness/attractiveness, in comparison with simple parental transmission, when populations are small. It is presumably these gains which led to its evolution in the first place.

In summary, the results for the modified and more realistic model confirm those for the simple parent-offspring transmission model. When cultural innovation processes take place and the results are passed on by a combination of vertical and oblique transmission, larger populations have a very major advantage over smaller ones. Quite simply, members of larger populations are on average both biologically fitter and more attractive as models for imitation, by virtue of the fact that the deleterious sampling effects present in small populations decline as population sizes increase. When populations are small, innovations which are less beneficial reproductively and less attractive to imitate are more likely to be maintained within them.

Finally, it is worth noting that there are two ways in which a population can be regarded as increasing. One is the obvious one of demographic growth in a local population. The other arises when an isolated population establishes contact with another population (cf. Peck 1996; also Neiman 1995). Both ways will produce the effects described above.

\section{Case-study: the emergence of modern human culture}

One major problem which may be illuminated by these results is the emergence of modern human culture in the last 50,000 years. In the case of Europe, Mellars (1996) notes the following characteristic features of the transition from Middle Palaeolithic (MP) to Upper Palaeolithic (UP):

- a shift in the technology of stone tool production towards the production of blades;
- rapid proliferation in the forms of stone tools;

- a proliferation of new forms of bone, antler and ivory tools;

- a much increased tempo of technological change;

- the appearance of a wide variety of forms of personal ornament;

- the appearance of the first musical instruments;

- the sudden appearance of complex forms of art.

The African Late Stone Age has been seen in a similar light as involving major innovations. It has been assumed until recently that these cultural changes are explicable in terms of the advent of the cognitive capacities of modern humans. The increasing evidence that modern humans originated in Africa between 200 and $100 \mathrm{ka}$ (see e.g. Lahr \& Foley 1998), however, has created a problem for this assumption. It has become apparent that a period of at least 50,000 and probably nearer 100,000 years elapsed between the time of origin of Homo sapiens and the features listed by Mellars. This situation is not easily explicable if modern human culture was somehow a straightforward consequence of modern biological/ cognitive capacities.

One possible way out of this problem is to suggest, as does Klein (1992; 1995), that some significant neural mutation took place after modern humans had developed, at some point much nearer in time to the cultural developments of the Upper Palaeolithic (Europe)/Late Stone Age (Africa). A major problem with this hypothesis, as Klein himself recognizes $(1995,188-9)$, is that it seems highly likely that by the time of the putative neural mutation $(c .55 \mathrm{ka})$ modern humans had already split into separate populations (see below), so we would have to suppose either this mutation occurred independently in different modern human lineages, or that there was a further world colonization wave by individuals bearing the mutation, for which we have no evidence.

It has also become increasingly clear that the association between modern humans and the UP/ LSA archaeological features regarded as characteristic of modern human culture is more complex than was initially believed (Foley \& Lahr 1997). Some of the features which have traditionally been regarded as part of the Upper Palaeolithic cultural complex do actually occur in Africa in MSA contexts not long after the origin of modern humans and in some cases associated with modern human fossils (Lahr 1996, 288; Foley \& Lahr 1997, 7; Lahr \& Foley 1998, 165), including bone harpoons (Brooks et al. 1995), the use of red ochre for colouring and drawing (Knight et al. 1995), and blade industries (Klein 1992); indeed, the 
widespread occurrence of pre-Upper Palaeolithic blade production has recently been strongly re-emphasized (Bar-Yosef \& Kuhn 1999). Moreover, all the earliest modern human fossils, whether in East Africa, southern Africa or the Near East are associated with standard MSA/MP industries (Foley \& Lahr 1997; Klein 1995).

The final complication to the standard picture is that the modern humans who colonized SouthEast Asia and Australia had no UP style blade industries at all (Foley \& Lahr 1997, 8), although they had the technology to make the sea crossing to Australia before c. 60,000 years ago (Lahr 1996, 292) and produced rock art and practised ritual burial at an early date.

In summary, it appears that modern humans originated in Africa well before 100,000 years ago, and that from an early date, at certain times and places, they showed evidence of modern capacities in the form of novel traits normally associated with the UP/LSA - including bone tools, use of colouring materials and blade industries. It also appears that there were no major cultural expansions until after 50,000 years ago, and that more, these were not uniform across the world when they did occur. While these expansions had biological prerequisites, in that they could not have occurred before the advent of modern cognitive capacities, their appearance does not have an explanation in terms of individual capacities. If this is the case, then the explanation must lie within some aspect of the social/cultural behaviour of human groups (cf. Lahr \& Foley 1998, 165). Following on from the simulation results presented above, it is suggested here that the cultural phenomena just outlined are explicable in terms of the human demographic patterns of the last 100,000 years.

\section{Palaeolithic demography}

Until recently Palaeolithic demography was extremely hypothetical to say the least, but the situation has now begun to change, as a variety of new lines of argument have begun to emerge. Best known of these is the advent of modern DNA-based anthropological genetics. Work by Rogers, Harpending and colleagues (Harpending et al. 1993; Sherry et al. 1994; Rogers \& Jorde 1995) on mtDNA mismatch distributions and other genetic evidence has led to the postulation of the so-called 'Weak Garden of Eden hypothesis' for modern human origins. This proposes that, after going through a bottleneck $c .130,000$ years ago, modern human populations separated from a common origin c. 100,000 years ago and underwent a period of growth some tens of thousands of years later, after going through a further series of local bottlenecks (Lahr \& Foley 1998, fig. 5). Views differ on whether the initial split and expansion led to populations moving out of Africa (Lahr \& Foley 1998) or was internal to Africa only, so that it was not until 60-80,000 years ago that a specific lineage of probably East African origin expanded out of Africa (Watson et al. 1997).

The implications of the various population bottleneck scenarios which have been put forward have recently been examined by Ambrose (1998). He suggests that the cause of the bottlenecks was a simultaneous worldwide volcanic winter generated by the Mt Toba eruption at $c .71 \mathrm{ka}(1998,632-5)$, immediately followed by the severe climatic conditions of OI stage 4. This would have led to a bottleneck of 10,000 years duration, with climatic release at 60,000 years ago making it possible for populations to start growing. Ambrose's demographic scenario is reproduced here as Figure 4.

Even if the Mt Toba eruption did not have the impact claimed, it is quite clear from recent reviews of the evidence (Lahr 1996; Lahr \& Foley 1998, 166) that the severe conditions of stage 4 led to major population fragmentation, including local extinctions of early modern human populations. Ambrose's discussion of the implications of this remains valid, regardless of whether the process was internal to Africa (Watson et al. 1997) or populations had already expanded beyond it.

In the light of the simulations described above, it can be seen that the demographic decline at around 70,000 years ago would have had major cultural implications. As local groups shrank or became extinct, cultural variation would have been lost. Once groups became small and isolated, the effect of small population size on the results of innovation processes, demonstrated in the simulations, would have become apparent: even if cultural novelty was being generated, levels of mean population fitness and attractiveness for imitation would have remained low.

Once the severe climatic conditions of stage 4 came to an end, however, local populations would have begun to increase. The simulations indicate that as this happened, cultural innovations would have become increasingly effective in leading to a major increase in combined mean fitness and mean attractiveness as a model to imitate in those populations. Deleterious sampling effects present in small populations decline as population sizes increase, so that advantageous cultural change is at least as much a consequence as a cause of increased population.

If, however, we associate the growth of cultural 


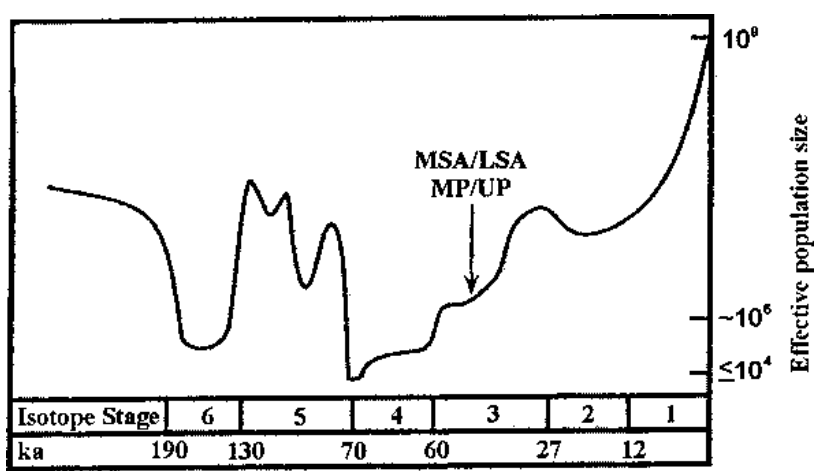

Figure 4. Estimates of modern human population size through the late Quaternary. (Redrawn from Ambrose 1998, fig. 5b.)

complexity with demographic 'bounce-back' after a long period of severe conditions, it is reasonable to ask why this did not happen even more quickly than it did. After all, even if we assume a relatively low growth rate of 0.5 per cent per year, after 4000 years the population would be moving up towards the one billion mark. That this did not happen as climatic conditions improved must be linked to low ceilings on carrying capacities, which were only gradually raised as a result of innovation processes of the kind modelled in this article.

Light is thrown on this question by Klein \& Cruz-Uribe's (1996) comparison of large bovid and seal exploitation at MSA and LSA sites in South Africa. The authors suggest that MSA people foraged less successfully than subsequent LSA populations in the same area. As far as large game were concerned, they favoured eland, which are relatively easy to drive, rather than buffalo, which all the environmental evidence suggests would have been more common but more difficult to exploit. LSA people did not prefer the eland, probably because they had weapons which enabled them to exploit other species more successfully. As a result, their population densities were higher than those of MSA populations, whose low densities are shown, among other things, by the large sizes of the tortoises and shellfish they collected, an indication that these prey populations were not under predation pressure. Klein \& CruzUribe (1996) also point out that MSA exploitation of seals is similar in pattern to that of hyenas, and did not take advantage of the seasonal abundance of 911 month old seals which was exploited by LSA and recent people.

The authors prefer to account for the changed pattern in terms of the emergence of fully modern human biological capacities. In the context of the present article an alternative explanation would be that successful innovations, developed in this region or elsewhere in the context of population increase, led to a rise in local carrying capacities and therefore populations, which would in turn have been productive of further successful innovation. Even if that is not the right explanation for this particular case, however, the study is important in indicating local population increase between MSA and LSA. More specifically it interprets low MSA populations stemming from low carrying capacities as a result of inefficient foraging behaviours, vulnerable to carrying a cultural 'drift load', on the one hand, and to the effects of climatic deterioration on their survival, on the other.

A recent study by Stiner et al. (2000) of small game exploitation during the Middle and Upper Palaeolithic in the Mediterranean regions comes to similar demographic conclusions. Examination of early MP faunal assemblages showed that up to 52 per cent of identifiable remains were of species such as tortoises with low replacement rates and therefore sensitive to heavy predation. The fact that the individuals of these species were of large body size, however, indicated that the prey populations were not under pressure, and therefore that human population densities must have been low, indicating small social groups, as the authors point out. Their study showed a first diminution in the size of one such such prey, the tortoise, in Israel, by at least 44,000 years ago but probably earlier; a size reduction which they interpreted as reflecting a major population growth pulse in the region at this time. Others subsequently occurred at later dates, both there and elsewhere in the Mediterranean. Stiner et al.'s (2000) results thus confirm the presence of population growth at a regional scale at precisely the time when rates of cultural change were increasing, in a region which has long been regarded as significant for the emergence of Eurasian UP cultural phenomena.

Finally, we should re-emphasize the point that the effects of actual demographic growth would have been magnified still further by the increasingly distant contacts for which we also have archaeological evidence in the form of raw material movements. More distant contacts imply a greater probability of being in contact with other people, which effectively enlarges the population on a scale proportional to the square of the distance radius. Thus, data from Europe indicate that the average greatest distance for the transfer of lithic raw material increased from around $50-60 \mathrm{~km}$ in the late Middle Palaeolithic to around $120 \mathrm{~km}$ in the Upper Palaeolithic (Féblot- 
Augustins 1997, quoted in Gamble \& Steele 1999; see also Blades 1999).

\section{Conclusions}

It now seems that on a broad scale the demographic picture of the last 100,000 years is one of decline to very small numbers around 70,000 years ago, followed by a major increase after 60,000 years ago, enhanced at least in some areas by increased rates of interaction from 40,000 years ago. This large-scale pattern is now being confirmed and amplified by local studies. The picture we have of the emergence of modern human culture suggests that there are occasional signs of its appearance before 70,000 years ago but that it does not become widely prevalent until after 50,000 years ago, long after the appearance of biologically modern humans. Thus there is a broad chronological correlation between major demographic increase and the emergence of modern human culture. The demonstration by the simulations that innovation produces low equilibrium fitness and low attractiveness values in small local populations, and values more than an order of magnitude greater in larger ones, suggests that demographic fluctuations may be the single most important factor in explaining how and why the emergence of modern human culture occurred when it did.

More generally, though, it appears possible that rates of successful technological innovation may have been correlated with population sizes and densities from the origins of hominin culture to the present. Is this the reason why handaxes barely changed for a million years?

\section{Acknowledgements}

I would like to thank Joel Peck for making his model available to me and for numerous discussions about the model itself, the way it should be presented and the interpretation of the results. I would also like to thank Lionel Barnett for modifying Peck's original computer program to cope with oblique transmission, and Mark Collard, Mark Lake and Chris Stringer for comments on an earlier version of the paper. Mark Collard's comments in particular led to a major re-write.

\section{Note}

1. Conceptually, Peck's model is straightforward and could in principle be handled analytically. In practice, however, working out its specific consequences for finite populations of various sizes with varying mutation rates can only be done by simulation, as Peck's papers in the population genetics literature demonstrate. This is therefore the procedure followed here since it is the specific consequences for finite populations which are of interest.

Stephen Shennan Institute of Archaeology University College London 31-34 Gordon Square

London

WC1H OPY

\section{References}

Ambrose, S., 1998. Late Pleistocene human population bottlenecks, volcanic winter, and differentiation of modern humans. Journal of Human Evolution 34, 62351.

Bar-Yosef, O. \& S.L. Kuhn, 1999. The big deal about blades: laminar technology and human evolution. American Anthropologist 101, 322-38.

Blades, B.S., 1999. Aurignacian lithic economy and early modern human mobility: new perspectives from classic sites in the Vézère valley of France. Journal of Human Evolution 37, 91-120.

Boyd, R. \& P.J. Richerson, 1985. Culture and the Evolutionary Process. Chicago (IL): University of Chicago Press.

Brooks, A.S., D.M. Helgren, J.S. Cramer, A. Franklin, W. Hornyak, J.M. Keating, R.G. Klein, W.J. Rink, H.P. Schwarcz, J.N.L. Smith, K. Stewart, N.E. Todd, J. Verniers \& J.E. Yellen, 1995. Dating and context of three Middle Stone Age sites with bone points in the Upper Semliki Valley, Zaire. Science 268, 548-53.

Cavalli-Sforza, L.L. \& M.W. Feldman, 1981. Cultural Transmission and Evolution: a Quantitative Approach. Princeton (NJ): Princeton University Press.

Féblot-Augustins, J., 1997. La circulation des matières premières au paléolithique. Liège: E.R.A.U.L.

Fisher, R.A., 1930. The Genetical Theory of Natural Selection. Oxford: Clarendon Press.

Foley, R.A. \& M.M. Lahr, 1997. Mode 3 technologies and the evolution of modern humans. Cambridge Archaeological Journal 7(1), 3-36.

Gamble, C. \& J. Steele, 1999. Hominid ranging patterns and dietary strategies, in Hominid Evolution: Lifestyles and Survival Strategies, ed. H. Ullrich. (Edition Archaea.) Germany: Leipzig.

Harpending, H., S.T. Sherry, A.R. Rogers \& M. Stoneking, 1993. The genetic structure of ancient human populations. Current Anthropology 34, 483-96.

Hewlett, B. \& L.L. Cavalli-Sforza, 1986. Cultural transmission among Aka pygmies. American Anthropologist $88,922-34$.

Klein, R.G., 1992. The archaeology of modern human origins. Evolutionary Anthropology 1, 5-14. 
Klein, R.G., 1995. Anatomy, behaviour and modern human origins. Journal of World Prehistory 9, 167-98.

Klein, R.G. \& K. Cruz-Uribe, 1996. Exploitation of large bovids and seals at Middle and Later Stone Age sites in South Africa. Journal of Human Evolution 31, 315-34.

Knight, C., C. Power \& I. Watts, 1995. The human symbolic revolution: a Darwinian account. Cambridge Archaeological Journal 5(1), 75-114.

Lahr, M.M., 1996. The Evolution of Modern Human Diversity. Cambridge: Cambridge University Press.

Lahr, M.M. \& R.A. Foley, 1998. Towards a theory of modern human origins: geography, demography and diversity in recent human evolution. Yearbook of Physical Anthropology 41, 137-76.

Mellars, P., 1996. The emergence of biologically modern populations in Europe: a social and cognitive 'revolution'?, in Evolution of Social Behaviour Patterns in Primates and Man, eds. W.G. Runciman, J. Maynard Smith \& R.I.M. Dunbar. Oxford: Clarendon Press, 179-202.

Neiman, F.D., 1995. Stylistic variation in evolutionary perspective - inferences from decorative diversity and inter-assemblage distance in Illinois Woodland ce- ramic assemblages. American Antiquity 60, 7-36.

Peck, J.R., 1996. Limited dispersal, deleterious mutations and the evolution of sex. Genetics 142, 1053-60.

Peck, J.R., G. Barreau \& S.C. Heath, 1997. Imperfect genes, Fisherian mutation and the evolution of sex. Genetics 145, 1171-99.

Rogers, A.R. \& L.B. Jorde, 1995. Genetic evidence on modern human origins. Human Biology 67, 1-36.

Shennan, S.J. \& J. Steele, 1999. Cultural learning in hominids: a behavioural ecological approach, in Mammalian Social Learning: Comparative and Ecological Perspectives, eds. H. Box \& K. Gibson. Cambridge: Cambridge University Press, 367-88.

Sherry, S.T., A.R. Rogers, H.C. Harpending, H. Soodyall, T. Jenkins \& M. Stoneking, 1994. Mismatch distributions of mtDNA reveal recent human population expansions. Human Biology 66, 761-75.

Stiner, M.C., N.D. Munro \& T.A. Surovell, 2000. The tortoise and the hare: small-game use, the broad-spectrum revolution and palaeolithic demography. Current Anthropology 41, 39-73.

Watson, E., P. Forster, M. Richards \& H-J. Bandelt, 1997. Mitochondrial footprints of human expansions in Africa. American Journal of Human Genetics 61, 691-704. 